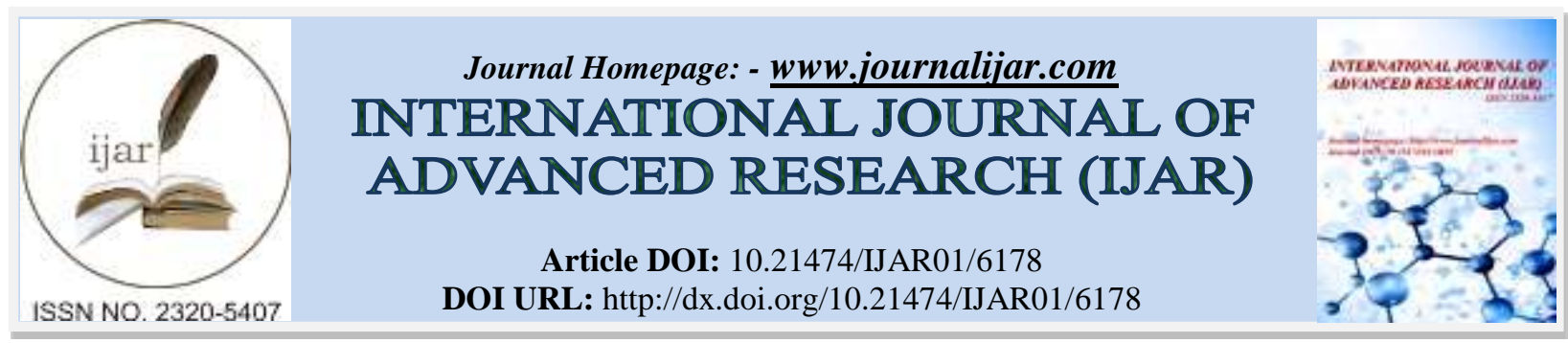

RESEARCH ARTICLE

\title{
ONCOSTATIN-M EXPRESSION IN ORAL SQUAMOUS CELL CARCINOMA.
}

\section{Noha Shaban Shams ${ }^{1}$, louloua Mohamed Fathy ${ }^{2}$ and Amal Hassan Abd-El Rahman ${ }^{2}$.}

1. Lecturer, Oral and Maxillofacial Pathology Department Faculty of Oral and Dental Medicine Ahram Canadian University.

2. Professor of Oral and Maxillofacial Pathology, Faculty of Oral and Dental Medicine, Cairo University.

3. Professor of Oral and Maxillofacial Pathology, Faculty of Oral and Dental Medicine, Cairo University.

\section{Manuscript Info}

\section{Manuscript History}

Received: 01 November 2017

Final Accepted: 03 December 2017

Published: January 2018

Key words:-

Oncostatin-m, oral squamous cell carcinoma, inflammatory cytokine.

\section{Abstract}

Background: Growth of malignant lesions is invariably associated with the presence of inflammatory cells at the primary site. It is hypothesized that tumor cells evade the growth inhibitory effects of cytokines present in the tumor microenvironment. Oncostatin -M $(\mathrm{OSM})$ is an inflammatory cytokine produced by macrophages and $\mathrm{T}$ lymphocytes as well as tumor cells, it inhibits the proliferation and induces differentiation of a number of types of tumors. This study aimed to assess OSM expression in oral squamous cell carcinoma.

Methods: Ex vivo study done on thirty (OSCC) cases obtained from paraffin blocks and ten normal epithelial tissues used as control. For each case immunohistochemical examination of OSM expression in different grades of OSCC was done.

Results: OSM immunohistochemical expression showed statistical significant difference between different grades of OSCC and also between them and control $(\mathrm{p}<0.0001)$.

Conclusion: This study found that OSM expression is inversely proportional with tumor differentiation suggesting that it may be used as biomarker in OSCC.

Copy Right, IJAR, 2018,. All rights reserved.

\section{Introduction:-}

Oral squamous cell carcinoma (OSCC) represents $95 \%$ of all forms of head and neck cancer, and during the past decade its incidence worldwide has increased by $50 \%{ }^{(5)}$. In Egypt 2008-2011, oral cancer in males was statistically represented as $0.23 \%$ in lip, $0.27 \%$ in tongue, $0.41 \%$ in mouth while in females; it was $0.21 \%$ in lip, $0.26 \%$ in tongue, $0.28 \%$ in mouth ${ }^{(13)}$.

Growing evidences designate that the carcinogenesis and progression of the tumor is determined by tumor cells as well as by tumor microenvironment (TME) as an integral aspect and essential area of cancerous tissues. The TME contains numerous types of cells, including fibroblasts, cancer-associated fibroblasts which are the most abundant cell type, endothelial cells and their precursors, pericytes, inflammatory cells, and antigen presenting cells, such as macrophages and dendritic cells. Cancer and inflammation are closely intertwined, and it is clear that invasion of normal tissue by malignancy generates a host inflammatory response to solid tissue tumor. Tumors that successfully expand must manipulate or navigate host responses. Both angiogenesis and extracellular matrix remodeling are required in the growth and metastatic potential of solid tumors ${ }^{(27)}$. 
It is now widely accepted that inadequately resolved chronic inflammation may increase the risk of cancer by providing bioactive molecules from cells infiltrating the tumor microenvironment. This includes cytokines; growth factors; chemokines that maintain a sustained proliferative rate; cell survival signals to avoid apoptosis; proangiogenic factors and extracellular matrix-modifying enzymes ${ }^{(9)}$.

Cytokines are low-molecular-weight proteins that mediate cell-to-cell communication, they bind to their receptors and trigger intracellular signaling events that result in the modulation of gene expression. Most cytokine receptors are comprised of a multi-subunit complex containing a unique and specific ligand binding subunit and a signal transducing subunit. Immune and stromal cells, such as fibroblasts and endothelial cells, synthesize them and they regulate proliferation, cell survival, differentiation, immune cell activation, cell migration, and death. Depending on the TME, cytokines can modulate an antitumoral response, but during chronic inflammation, they can also induce cell transformation and malignancy, conditional on the balance of pro- and anti-inflammatory cytokines, their relative concentrations, cytokine receptor expression content, and the activation state of surrounding cells ${ }^{(26)}$.

Oncostatin- M (OSM) was first purified and biochemically characterized on the basis of its anti-proliferative activity on the A375 human melanoma cell lines in vitro ${ }^{(25)}$. Its name was coined based on this inhibitory function on A375 and other melanoma cell lines. The crystal structure of human OSM reveals a typical hematopoietic cytokine topology with up-up-down-down four-helix bundle with specific site 2 and site 3 epitopes predicted to interact with receptor chains on cell surfaces ${ }^{(16)}$.

OSM was found to interact with receptor complexes that included the cell signaling molecule glycoprotein 130 (gp130) and thus has been grouped with this gp130 family of cytokines that include interleukin-6 (IL-6), leukemia inhibitor factor (LIF), interleukin-11 (IL-11), cardiotrophin-1 (CT-1), ciliary neurotrophic factor (CNTF), neurotrophin-1/BSF-3), IL-27 ${ }^{\text {(21) }}$.

In contradiction of other members of this family, OSM binds gp130 with low affinity and as such has slight to no biological activity unless a second receptor chain is recruited, either the LIFR $\alpha$ or the specific OSM Receptor-beta chain $(\mathrm{OSMR} \beta)^{(15)}$.

Individual cell populations that express OSM comprise activated macrophages, monocytes, $\mathrm{T}$ cells, and dendritic cells ${ }^{(24)}$. It could be released by neutrophils from preformed stores and these cells could also synthesize OSM upon stimulation by granulocyte colony stimulating factor ${ }^{(5)}$.

Along with OSM role in inflammation, an impending role in inhibiting cellular proliferation was explored for a while, far along its functions have been further explored in various cancers and tumor systems. Researches done on OSCC are relatively limited thus this study anticipated to explore its expression on protein level ${ }^{(23)}$.

\section{Materials and Methods:-}

A total of 30 specimens of OSCC and 10 normal epithelial tissues were retrieved from the archival paraffin blocks from the pathological files of the Department of Oral and Maxillofacial Pathology, Faculty of Oral and Dental Medicine, Department of General Pathology, Faculty of Medicine Cairo University and from Maxillofacial Surgery Department of Naser Institute. Normal tissues were obtained from normal gingival tissues from crown lengthening procedures. $65 \%$ of patients were males and most cases were obtained from tongue, floor of the mouth and palate. This work was approved by ethics committee of Cairo University

Tissue sections were stained with Hematoxylin and Eosin stain and were rediagnosed to confirm the previously made diagnosis. Other consecutive sections were cut on the positively charged glass slides (Opti-Plus, Biogenex Laboratory, USA) for imunohistochemical staining.

\section{Methods:-}

Formalin-fixed, paraffin-embedded 5-m-thick sections from a representative block in each case were subjected to OSM rabbit polycolonal antibody (Cat.No.NBP1-87768, Bioss, SNF MEDICAL, USA).. Immunohistochemical staining was performed using the The universal immunostaining detection kit (Dako Cytomation Envision+ Dual link system, Peroxidase (DAB+) Code K4065, Carpinteria, CA, USA) first Epitope retrieval: Citrate Buffer Epitope Retrieval method was used in this technique The tissue were incubated for I hour at room tempreture in primary antibody. To confirm the specificity of the primary antibodies, negative control slides were run with every batch 
using serum buffer substrate at the same concentration as that of the primary antibodies then Secondary antibody in the form of biotinylated horse Anti-Mouse $\operatorname{IgG}$ diluted in secondary antibody dilution was added. Finally chromogen/substrate sections were incubated in DAB peroxidase substrate solution followed by counter stain.

In order to provide more précised data on OSM immunoexpression in OSCC, image analyzer was used. The data was obtained using the software (SIS, Germany), and by using the image analyzer, five specific fields showing maximum positivity of OSM expression were used in each case; the mean of five values of each slide was calculated and statistically analyzed. For each field of immunostained section, a measuring frame of area 1920000 pixels and then the area $\%$ was calculated. The color of immunostaining was automatically selected according to the positive control sections, converted into grey color then masked the red and white to allow automatic measurements by the computer system. Data obtained were subjected to statistical analysis using (ANOVA) test.

\section{Results:-}

Normal epithelial tissues showed faint cytoplasmic expression of OSM among epithelial cells in addition to positive expression in connective tissue stroma especially collagen fibers (Fig.1).

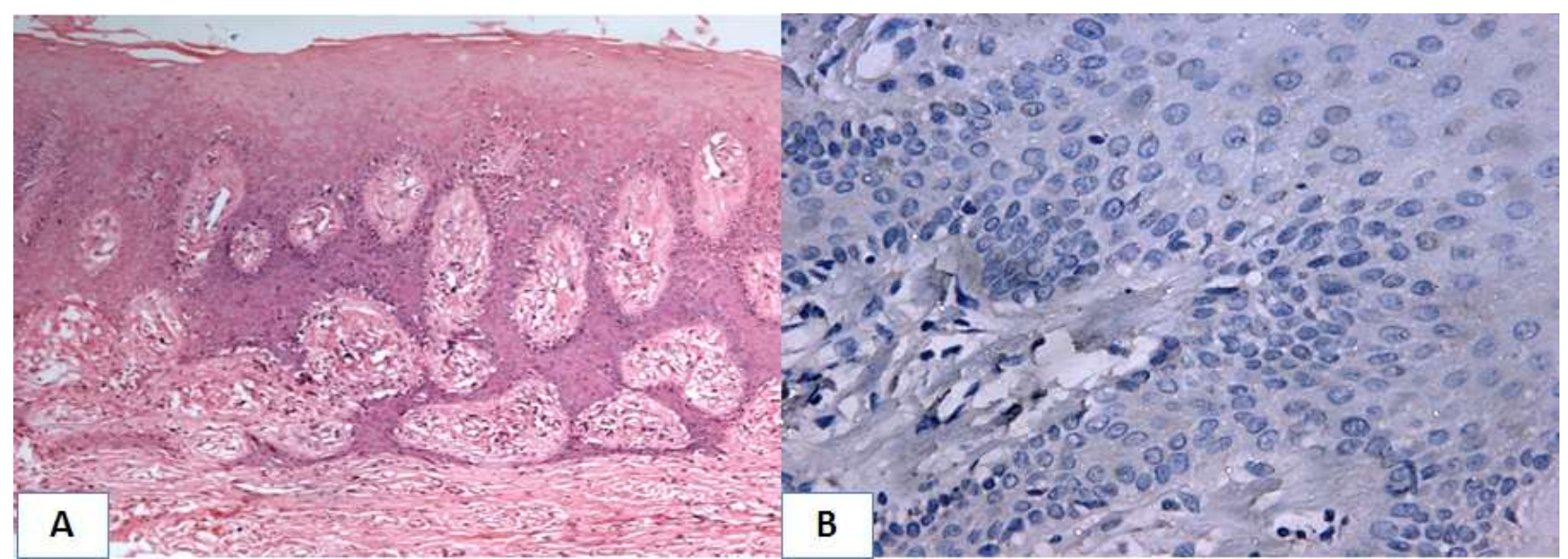

Fig.1 (a):- photomicrograph of normal gingival stratified squamous epithelium (H\&E, x 100), (b) photomicrograph of normal gingival stratified squamous epithelium showing faint cytoplasmic immunopositivity of OSM in epithelial and stromal cells (OSM, $\mathbf{x}$ 400).

In well differentiated OSCC epithelial cells in all specimens showed higher homogenous cytoplasmic OSM expression compared to normal as well as in keratin pearls, while stromal cells showed positive expression in collagen fibers along with tumor associated inflammatory cells especially tumor associated macrophages (Fig.2).

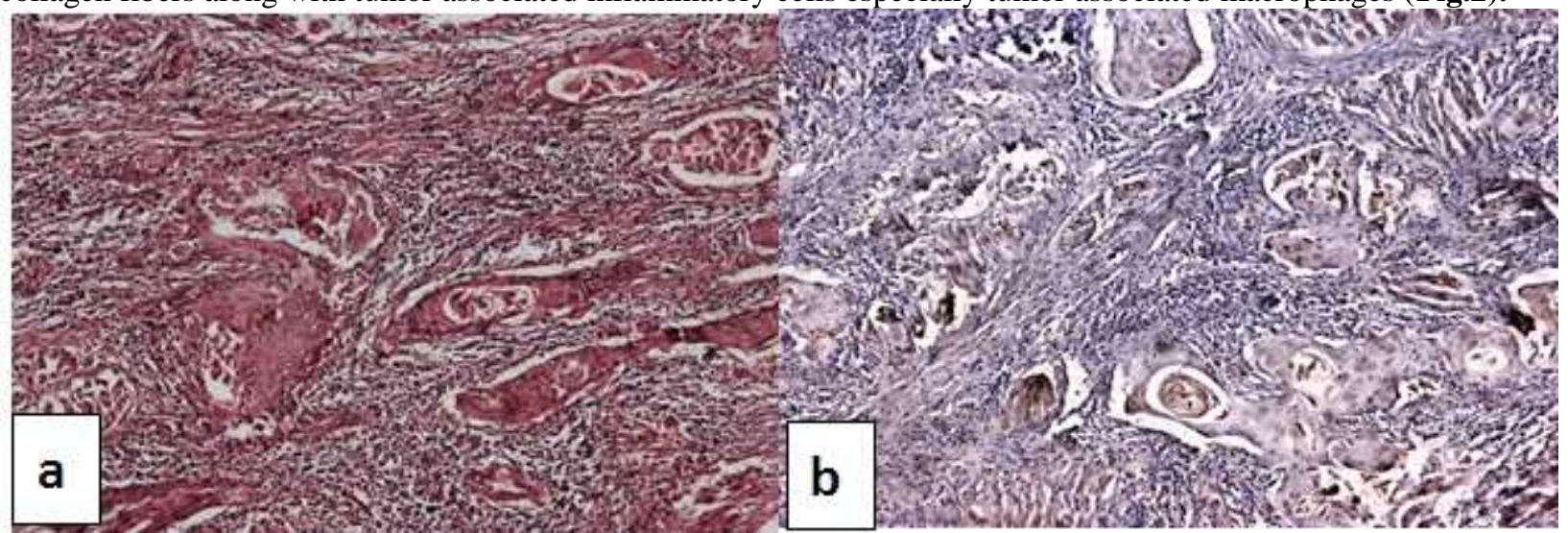

Fig: (3):- (a) photomicrograph of well differentiated OSCC showing numerous cell nests and keratin pearls (H\&E, $\mathbf{x}$ 100). (b): photomicrograph of the previous figure showing cytoplasmic OSM immunostaining in neoplastic cells and keratin pearls (OSM x 100). 
Eight specimens of moderately differentiated group showed stronger homogenous cytoplasmic OSM expression in both neoplastic epithelial cells as well as stromal cells more than what was shown in normal and well differentiated ones. While two specimens showed unnoticeable expression in neoplastic cells with more intense expression in stromal cells and tumor associated macrophages (Fig3).

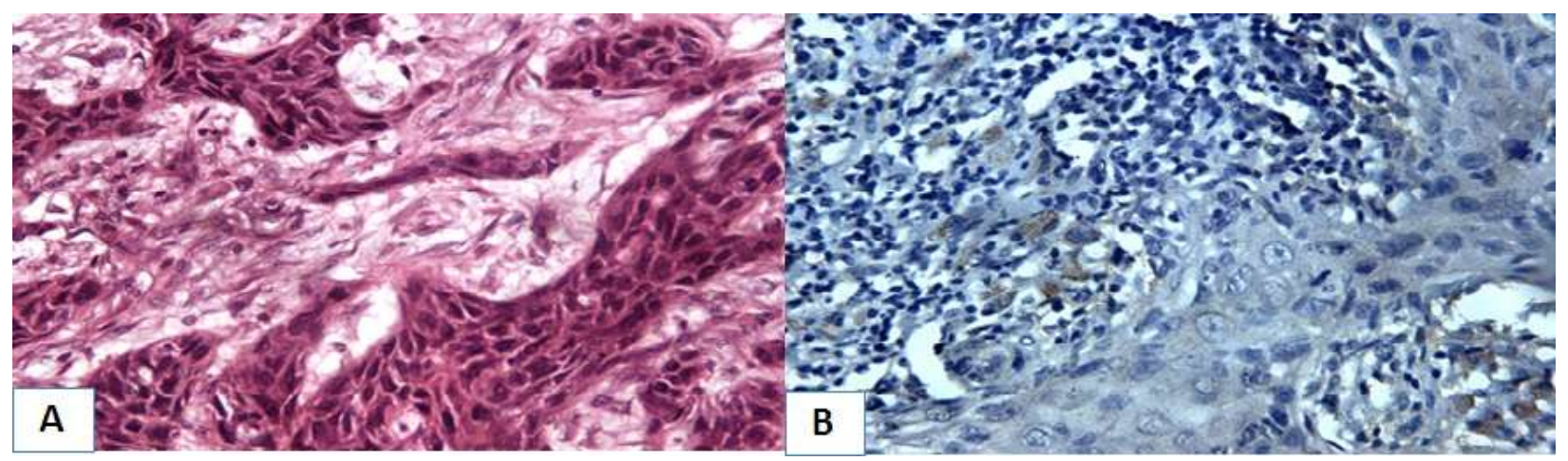

Fig (3):- (a) photomicrograph of moderately differentiated OSCC showing islands of tumor cells with few keratin pearls (H\&E x 400). (b): photomicrograph of previous figure showing an almost negative OSM immunostaining in neoplastic cells and stromal cells (OSM, $\mathbf{x} 400)$.

Finally, the most intense cytoplasmic OSM expression was shown in neoplastic cells of poorly differentiated OSCC and their tumor associated stromal cells, collagen fibers and inflammatory (Fig.4).

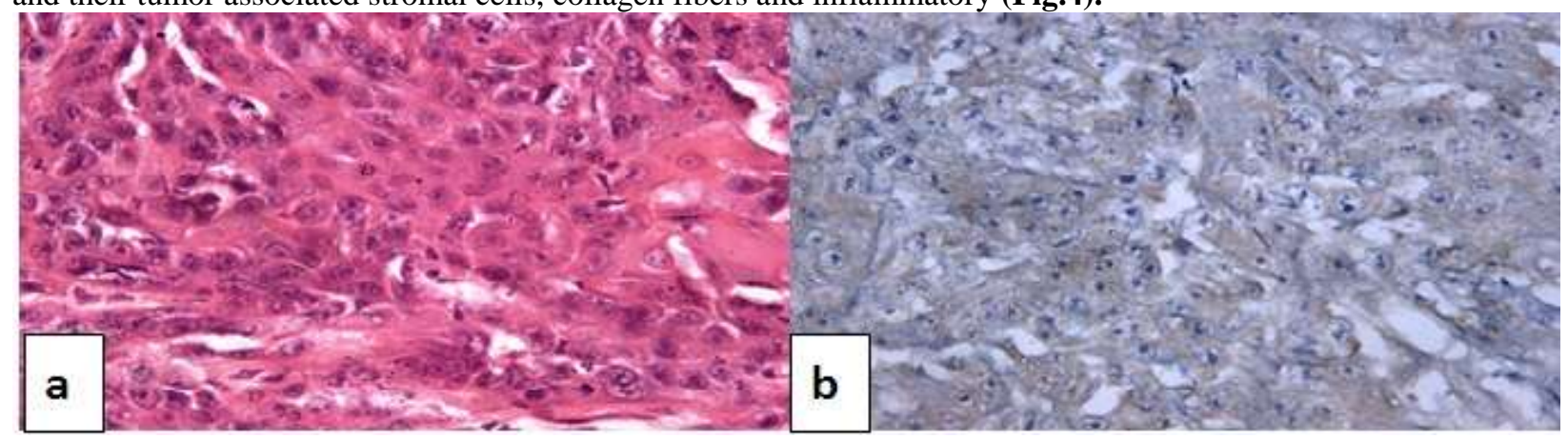

Fig (4):- (a): photomicrograph of poorly differentiated OSCC showing sheets of undifferentiated neoplastic cells (H\&E, x 400). (b): photomicrograph of the previous figure showing homogenous intense cytoplasmic OSM immunostaining in neoplastic cells (OSM, $\mathbf{x} 400)$.

Statistically, within the different grades of OSCC, The greatest mean value was recorded in poorly differentiated SCC, with the least value recorded in well- differentiated OSCC. One way analysis of variance (ANOVA) test revealed that the difference between different grades was statistically significant $(\mathrm{P}<0.0001)$. Tukey's post hoc test revealed no significant difference between well and moderately differentiated SCC. Moreover, the mean value recorded in control was significantly lower than all grades of OSCC $(\mathrm{P}<0.0001)$ Fig. (5). 


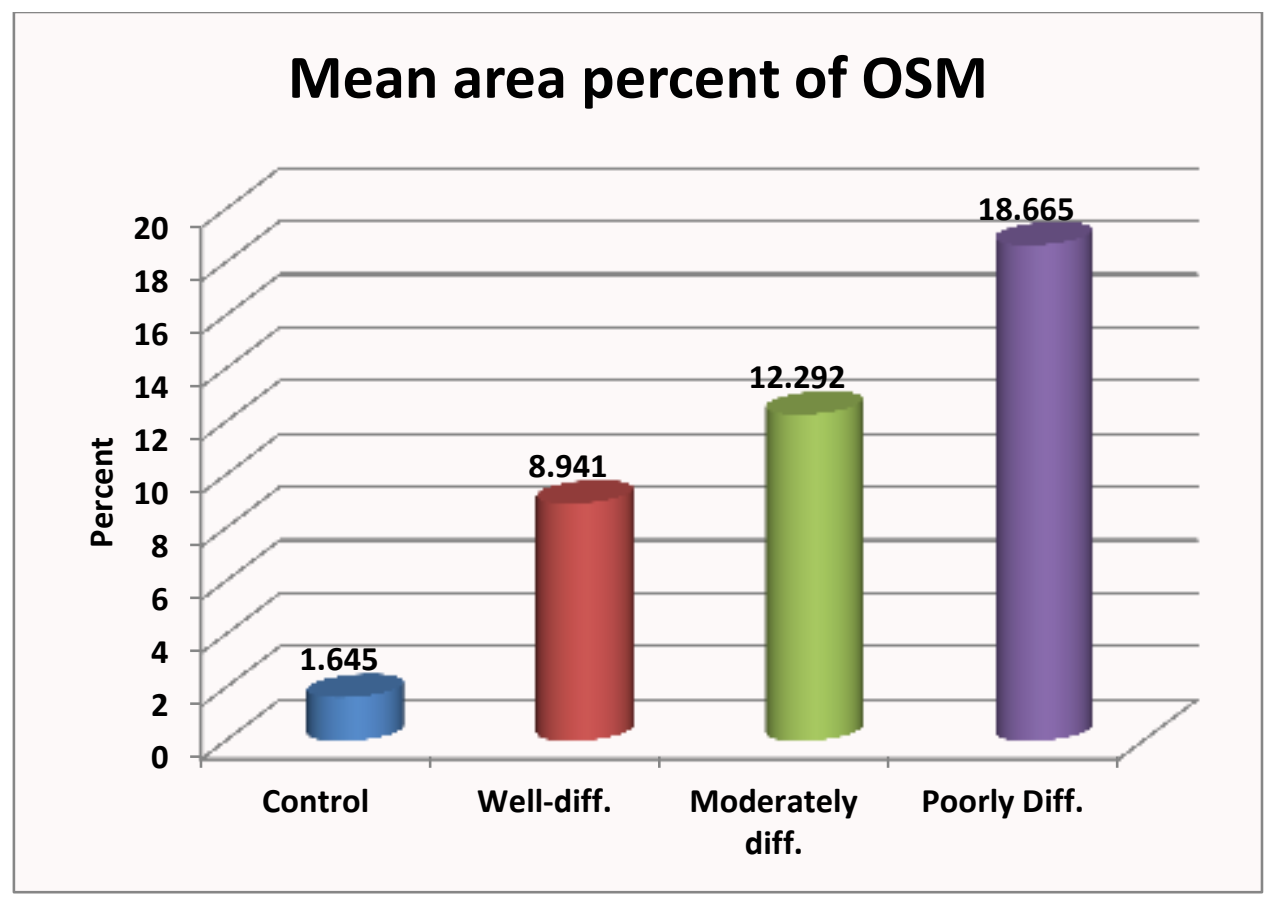

Fig. (5):- Column chart showing mean area percent of OSM immunoexpression

\section{Discussion:-}

It was found that expression of OSM has remarkable roles in inflammation and tumorgenesis as modulator of cellular proliferation, epithelial mesynchymal transition, angiogenesis and metastases, thus further investigations of its expression on protein levels was required ${ }^{(18)}$.

In the current study, all OSCC specimens showed variable homogenous cytoplasmic OSM immunoexpression in tumor cells. This finding may be attributed to endogenous synthesis of OSM by the tumor cells or it may denote a bound form of OSM and its receptor.Although OSM is predominantly formed by activated T lymphocytes and macrophages, the possibility that OSM is produced by tumor cells was previously supported by studies of OSM synthesis in Kaposi's sarcoma ${ }^{(\mathbf{1})}$, in OSCC ${ }^{(\mathbf{2 2})}$, in prostate carcinoma ${ }^{(\mathbf{1 9})}$, in breast cancer ${ }^{(\mathbf{7}) \text {, in esophageal SCC }}{ }^{(\mathbf{1 2})}$ and lastly in OSCC Chuerduangphui et al suggested that keratinocyte growth factor can stimulate OSM expression in tumor cells ${ }^{(2)}$.

We detected that the most pronounced OSM immunoexpression was recorded in poorly differentiated OSCC while the least expression was found in well differentiated ones where the difference was statistically significant indicating its role in tumor progression. Kozlowska et al explained OSM role in progression where they reported a potential role for OSM to inhibit the activity of metalloproteinase inhibitor which explained the role of this cytokine in neoplastic metastases ${ }^{(13)}$. Furthermore, studies done on the role of OSM in breast cancer have shown that a complex set of activities are likely involved. Snyder et al, have shown that OSM induces a STAT3-dependent mechanism and thus contributes to a migratory and invasive phenotype of breast cancer cells by inhibiting apoptosis through induction of bcl-2 or TGF-beta ${ }^{(20)}$. Furthermore, STAT-3 whenever it is activated by gp130 it induces activation of c-myc gene and up-regulation of cyclins D1, D2, D3, which are responsible for the G1 to S cell-cycle progression signals that are elicited by STAT3 that has been activated by gp130 ${ }^{(10)}$. In addition, OSM can induce EMT and cancer stem cell-like features in breast cancer cells in vitro ${ }^{(8,24)}$.

As regards the stromal immunoreactivity of OSM, it was identified in all specimens in fibroblasts and collagen fibers, this was explained by Somasundaram et al who reported that human OSM has been found to bind to collagens I, III, and IV in vitro ${ }^{(21)}$.

Only two specimens out of thirty OSCC, where the neoplastic cells showed faint OSM immunoexpression while the stromal expression showed intense immunoexpression. Comparable results were observed by Tran et al, they 
explained this indirectly proportional relationship by the possibility of presence of negative feedback mechanism between the tumor cells and the surrounding infiltrative macrophages ${ }^{(22) .}$ Inefficient OSM protein expression in neoplastic cells was also explained by Radtke et al who suggested the presence of abnormalities in various stages of OSMR $\beta$ production and activation ${ }^{(17)}$, furthermore, Lacreusetteet al., 2007 in their study on melanoma, identified epigenetic modifications in the OSMR $\beta$ promoter as histone deacetylation ${ }^{(14)}$. Another potential mechanism was proposed by Kausar et al who detected a spliced variant encoding a soluble form of OSMR $\beta$ evident in esophageal cancer tissue which binds with OSM and thus acts as neutralizing receptor for OSM ${ }^{(12)}$. This hypothesis was also confirmed by Diveu et al in their study on glioblastoma ${ }^{(4)}$.

\section{Conclusion:-}

It might be concluded that, OSM production is modulated through stromal and neoplastic cells which in turn have an important role in OSCC progression and hypothesizes the possibility of its use as biomarker.

\section{References:-}

1. Cai, J., P. S. Gill, R. Masood, P. Chandrasoma, B. Jung, R. E. Law, and S. F. Radka. "Oncostatin-M is an autocrine growth factor in Kaposi's sarcoma." The American journal of pathology 145, no. 1 (1994): 74.

2. Chuerduangphui, Jureeporn, Chamsai Pientong, Ponlatham Chaiyarit, Natcha Patarapadungkit, Apinya Chotiyano, Bunkerd Kongyingyoes, Supannee Promthet, Piyawut Swangphon, Weerayut Wongjampa, and Tipaya Ekalaksananan. "Effect of human papillomavirus 16 oncoproteins on oncostatin M upregulation in oral squamous cell carcinoma." Medical Oncology 33, no. 8 (2016): 1-11.

3. Dissanayaka, Waruna Lakmal, Gayani Pitiyage, Pallegoda Vithanage Ranjith Kumarasiri, Rubasinha Liyanage Pemith Ranura Liyanage, Kumara Dayanath Dias, and Wanninayake Mudiyanselage Tilakaratne. "Clinical and histopathologic parameters in survival of oral squamous cell carcinoma." Oral surgery, oral medicine, oral pathology and oral radiology 113, no. 4 (2012): 518-525.

4. Diveu, Caroline, Emilie Venereau, Josy Froger, Elisa Ravon, Linda Grimaud, François Rousseau, Sylvie Chevalier, and Hugues Gascan. "Molecular and functional characterization of a soluble form of oncostatin M/interleukin-31 shared receptor." Journal of Biological Chemistry 281, no. 48 (2006): 36673-36682.

5. Elbjeirami, Wafa M., Elizabeth M. Donnachie, Alan R. Burns, and C. Wayne Smith. "Endothelium-derived GM-CSF influences expression of oncostatin M." American Journal of Physiology-Cell Physiology 301, no. 4 (2011): C947-C953.

6. Fossey, Stacey L., Misty D. Bear, William C. Kisseberth, Michael Pennell, and Cheryl A. London. "Oncostatin M promotes STAT3 activation, VEGF production, and invasion in osteosarcoma cell lines." BMC cancer 11, no. 1 (2011): 125.

7. García-Tunón, Ignacio, Monica Ricote, Antonio Ruiz, Benito Fraile, Ricardo Paniagua, and Mar Royuela. "OSM, LIF, its receptors, and its relationship with the malignance in human breast carcinoma (in situ and in infiltrative)." Cancer investigation 26, no. 3 (2008): 222-229.

8. Guo, L., C. Chen, M. Shi, F. Wang, X. Chen, D. Diao, M. Hu, M. Yu, L. Qian, and N. Guo. "Stat3-coordinated Lin-28-let-7-HMGA2 and miR-200-ZEB1 circuits initiate and maintain oncostatin M-driven epithelialmesenchymal transition." Oncogene 32, no. 45 (2013): 5272-5282.

9. Hanahan, Douglas, and Robert A. Weinberg. "Hallmarks of cancer: the next generation." cell 144, no. 5 (2011): 646-674.

10. Hirano, Toshio, Katsuhiko Ishihara, and Masahiko Hibi. "Roles of STAT3 in mediating the cell growth, differentiation and survival signals relayed through the IL-6 family of cytokine receptors." Oncogene 19, no. 21 (2000): 2548.

11. Ibrahim, Amal S., Hussein M. Khaled, Nabiel NH Mikhail, Hoda Baraka, and Hossam Kamel. "Cancer incidence in Egypt: results of the national population-based cancer registry program." Journal of cancer epidemiology 2014 (2014).

12. Kausar, Tasneem, Rinu Sharma, Md Raghibul Hasan, Anoop Saraya, Tushar K. Chattopadhyay, Siddartha Datta Gupta, and Ranju Ralhan. "Overexpression of a splice variant of oncostatin M receptor beta in human esophageal squamous carcinoma." Cellular Oncology 34, no. 3 (2011): 177-187.

13. Kozlowska, K., Małgorzata Zarzeczna, and Mirosława Cichorek. "A comparative study on oncostatin M secretion by transplantable melanoma cells with regard to their biological properties and progression." FOLIA MORPHOLOGICA-WARSZAWA-ENGLISH EDITION- 60, no. 2 (2001): 85-90. 
14. Lacreusette, A., J. M. Nguyen, M. C. Pandolfino, A. Khammari, B. Dreno, Y. Jacques, A. Godard, and F. Blanchard. "Loss of oncostatin M receptor $\beta$ in metastatic melanoma cells." Oncogene 26, no. 6 (2007): 881892.

15. O'hara, K. A., M-A. Kedda, P. J. Thompson, and D. A. Knight. "Oncostatin M: an interleukin-6-like cytokine relevant to airway remodelling and the pathogenesis of asthma." Clinical \& Experimental Allergy 33, no. 8 (2003): 1026-1032.

16. Plun-Favreau, Hélène, David Perret, Caroline Diveu, Josy Froger, Sylvie Chevalier, Eric Lelièvre, Hugues Gascan, and Marie Chabbert. "Leukemia inhibitory factor (LIF), cardiotrophin-1, and oncostatin M share structural binding determinants in the immunoglobulin-like domain of LIF receptor." Journal of Biological Chemistry 278, no. 29 (2003): 27169-27179.

17. Radtke, Simone, Heike M. Hermanns, Claude Haan, Hildegard Schmitz-Van de Leur, Hugues Gascan, Peter C. Heinrich, and Iris Behrmann. "Novel role of Janus kinase 1 in the regulation of oncostatin M receptor surface expression." Journal of Biological Chemistry 277, no. 13 (2002): 11297-11305.

18. Richards, Carl D. "The enigmatic cytokine oncostatin M and roles in disease." ISRN inflammation 2013.

19. Royuela, Mar, Monica Ricote, Melanie S. Parsons, Ignacio García-Tuñón, Ricardo Paniagua, and Maria P. De Miguel. "Immunohistochemical analysis of the IL-6 family of cytokines and their receptors in benign, hyperplasic, and malignant human prostate." The Journal of pathology 202, no. 1 (2004): 41-49.

20. Snyder, Marylynn, Xin-Yun Huang, and J. Jillian Zhang. "Signal transducers and activators of transcription 3 (STAT3) directly regulates cytokine-induced fascin expression and is required for breast cancer cell migration." Journal of Biological Chemistry 286, no. 45 (2011): 38886-38893

21. Somasundaram, Rajan, Martin Ruehl, Benjamin Schaefer, Monika Schmid, Renate Ackermann, Ernst O. Riecken, Martin Zeitz, and Detlef Schuppan. "Interstitial collagens I, III, and VI sequester and modulate the multifunctional cytokine oncostatin M." Journal of Biological Chemistry 277, no. 5 (2002): 3242-3246.

22. Tran, Tien-Anh, Jeffrey S. Ross, Christine E. Sheehan, and J. Andrew Carlson. "Comparison of oncostatin M expression in keratoacanthoma and squamous cell carcinoma." Modern Pathology 13, no. 4 (2000): $427-432$.

23. Wallace, Philip M., John F. MacMaster, Katherine A. Rouleau, T. Joseph Brown, James K. Loy, Karen L. Donaldson, and Alan F. Wahl. "Regulation of inflammatory responses by oncostatin M." The Journal of Immunology 162, no. 9 (1999): 5547-5555.

24. West, Nathan R., Leigh C. Murphy, and Peter H. Watson. "Oncostatin M suppresses oestrogen receptor- $\alpha$ expression and is associated with poor outcome in human breast cancer." Endocrine-related cancer 19, no. 2 (2012): 181-195.

25. Zarling, Joyce M., Mohammed Shoyab, Hans Marquardt, Marcia B. Hanson, Mario N. Lioubin, and George J. Todaro. "Oncostatin M: a growth regulator produced by differentiated histiocytic lymphoma cells." Proceedings of the National Academy of Sciences 83, no. 24 (1986): 9739-9743.

26. Zamarron B.F., Chen W. "Dual roles of immune cells and their factors in cancer development and progression" International Journal of Biological Sciences, 2011; vol. 7, no. 5, pp. 651-658.

27. Zhang, Jing, and Jinsong Liu. "Tumor stroma as targets for cancer therapy." Pharmacology \& therapeutics 137 , no. 2 (2013): 200-215. 